\title{
Effect of synbiotic supplementation on children with atopic dermatitis: an observational prospective study
}

\author{
M. Dolores Ibáñez ${ }^{1,2,3} \cdot$ Pablo Rodríguez del Río ${ }^{1,2,3} \cdot$ Diego González-Segura Alsina $^{4} \cdot$ Vicenç Villegas Iglesias ${ }^{4}$
}

Received: 10 April 2018 / Revised: 12 September 2018 / Accepted: 12 September 2018 / Published online: 26 September 2018

(C) The Author(s) 2018

\begin{abstract}
The objective of this observational single-cohort prospective study was to assess the effect of synbiotic supplementation for 8 weeks in children with atopic dermatitis (AD). The synbiotic product contained Lactobacillus casei, Bifidobacterium lactis, Lactobacillus rhamnosus, Lactobacillus plantarum, fructooligosaccharide, galactooligosaccharide, and biotin. Patients were examined at baseline and at 8 weeks. Effectiveness of treatment was assessed with the Scoring Atopic Dermatitis (SCORAD) index. A total of 320 children (mean age 5.1 years, range $0-12$ years) were included. The mean (SD) SCORAD index decreased from 45.5 (15.5) at baseline to $19.4(14.6)$ at the end of treatment $(P<0.001)$, VAS score for pruritus decreased from $5.7(2.2)$ to 2.3 (2.2) $(P<0.001)$, and VAS score for sleep decreased from $3.1(2.5)$ to $1.1(1.8)(P<0.001)$. Percentage of children with moderate-severe disease decreased from $92.4 \%$ at baseline to $28.1 \%$ at week 8 . In the multiple linear regression analysis, higher baseline SCORAD index (OR 0.51; 95\% CI 0.41-0.61) and higher adherence (OR 7.29; 95\% CI 1.85-12.73) were significantly associated with greater decrease in SCORAD index.

Conclusion: Supplementation with the multistrain synbiotic product may improve AD in children.

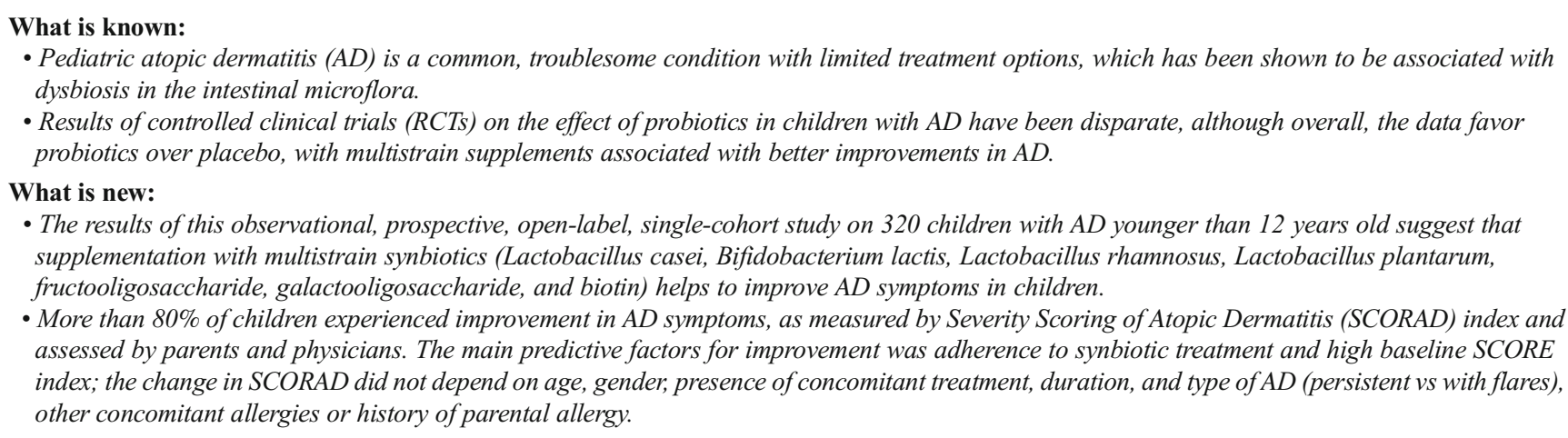

Keywords Pediatric atopic dermatitis · Probiotics · Synbiotics $\cdot$ Lactobacillus casei $\cdot$ Bifidobacterium lactis $\cdot$ Lactobacillus rhamnosus $\cdot$ Lactobacillus plantarum

Communicated by Mario Bianchetti

M. Dolores Ibáñez

mibanezs@salud.madrid.org

Pablo Rodríguez del Río

prrio@yahoo.es

Diego González-Segura Alsina

d.gonzalez@chiesi.com

Vicenç Villegas Iglesias

v.villegas@chiesi.com
1 Allergy Department and Biomedical Research Foundation (FIB), Hospital Infantil Universitario Niño Jesús, Avda. Menéndez Pelayo 56, E-28009 Madrid, Spain

2 Health Research Institute, IIS Princesa, Madrid, Spain

3 Allergy National Network (ARADyAL), Institute Carlos III, Madrid, Spain

4 Medical department, Chiesi Spain S.A.U., Barcelona, Spain 


\begin{tabular}{ll}
\multicolumn{2}{l}{ Abbreviations } \\
AD & Atopic dermatitis \\
CFU & Colony-forming units \\
CI & Confidence interval \\
ITT & Intention to treat \\
OR & Odds ratio \\
PP & Per protocol \\
RCT & Randomized controlled trials \\
SCORAD & Severity Scoring of Atopic Dermatitis \\
SD & Standard deviation \\
VAS & Visual analogue scale
\end{tabular}

\section{Introduction}

Atopic dermatitis $(\mathrm{AD})$ is a common chronic, relapsing inflammatory allergic disease with highly pruritic skin lesions, particularly prevalent in children. It usually starts in the first 5 years of life and often has a profound negative effect on the quality of life of patients and their families [1]. AD affects up to $20 \%$ of children and $3 \%$ of adults, with the prevalence increasing globally [18].

The successful management of AD includes hydration, restoration of the skin barrier, control of skin inflammation, and treatment of secondary infections $[10,14]$. Topical corticosteroids remain the first-line medical treatment for the control of symptoms, but relapses are common [20] and adverse effects limit their chronic use [11]. Calcineurin inhibitors are sometimes effective in reducing inflammation and help spare the use of topical steroids [5].

Synbiotics have been defined as combinations of pre- and probiotics with a synergistic action on human health [8]. Prebiotics are food components that induce the growth or activity of probiotics, which are living organisms that, when administered in adequate amounts (at least $10^{9}$ colonyforming units $[\mathrm{CFU}]$ ), can be beneficial in the treatment of various conditions. Widely known probiotics such as Bifidobacteria and Lactobacilli have been identified as key components for proper immune system stimulation and homeostasis of the gastrointestinal tract microenvironment [7, 21]. Dysbiosis with increased levels of Clostridium and low levels of Bifidobacterium species in the intestinal microbiota has been observed in atopic children and was speculated to contribute to inflammation in $\mathrm{AD}$ [13]. Balancing the gut microflora may improve gut barrier function and reduce the production of proinflammatory cytokines. In addition, several in vitro and clinical studies showed that consumption of probiotics suppressed Th2 response and shifted Th1/Th2 balance towards Th1 response [16, 17]. Recently, modulation of microbiota to promote clinical improvement in pediatric patients with $\mathrm{AD}$ has been a focus of increasing interest. Metaanalyses of randomized controlled trials have shown that multistrain pro- and synbiotics are of benefit for the prevention and treatment of AD in children $[2-4,6]$.

The main objective of this prospective observational study was to assess the effect of a multistrain synbiotic supplement containing Lactobacillus casei, Bifidobacterium lactis, Lactobacillus rhamnosus, and Lactobacillus plantarum plus oligosaccharides and biotin for 8 weeks in children with AD. Treatment tolerance was also evaluated.

\section{Methods}

\section{Study design and setting}

This observational, prospective, multicenter study was carried out in the outpatient pediatric and allergology clinics throughout Spain in daily practice conditions. The study was conducted in accordance with the Declaration of Helsinki (7th revision), the Spanish regulations on observational studies (Order SAS 3470/2009) and Spanish personal data protection law (Law 15/1999). The study protocol was approved by the Ethics Committee of Hospital Infantil Universitario Niño Jesús, Madrid, Spain. Parents or legal representatives of all patients gave written informed consent before inclusion. All data were anonymized.

\section{Study population}

The study population consisted of children $<12$ years old, diagnosed with $\mathrm{AD}$ who presented with active eczema at the time of consultation at primary care centers. Exclusion criteria were allergy or intolerance to probiotics or excipients of the synbiotic product and presence of any severe disease other than $\mathrm{AD}$ as well as other dermatological diseases which could interfere with assessment of AD skin lesions. Patients were recruited between April 1 and August 31, 2016.

\section{Treatment and study procedures}

The composition of the synbiotic product $(1 \mathrm{~g})$ was as follows: L. casei CBT LC5 $4 \times 10^{9} \mathrm{CFU}, B$. lactis CBT BL3 $2 \times$ $10^{9} \mathrm{CFU}$, L. rhamnosus CBT LR5 $2 \times 10^{9} \mathrm{CFU}$, L. plantarum CBT LP3 $2 \times 10^{9} \mathrm{CFU}$, biotin $7.5 \mathrm{mg}$, fructooligosaccharide $171.75 \mathrm{mg}$, and galactooligosaccharide $100 \mathrm{~g}$ (Produo® Derma, Chiesi España, S.A.U., L'Hospitalet de Llobregat, Barcelona, Spain). In this product, the probiotics were $L$. casei, L. lactis, L. rhamnosus and L. plantarum, while the prebiotics were fructooligosaccharide and galactooligosaccharide. A dose of $1 \mathrm{~g}$ ( 1 stick) twice a day was administered orally or dissolved in liquids or mixed with food. Treatment duration was 8 weeks.

The main study variable was changed in the Severity Scoring of Atopic Dermatitis (SCORAD) index [22]. 
Secondary variables were as follows: (1) demographic data (age, sex, parental education level); (2) profile of parental atopy ( $\mathrm{AD}$, food, drug, and respiratory allergy), living with pets, respiratory allergy, food allergy, drug allergy, duration of $\mathrm{AD}$, age at onset, persistent (constant presence of symptoms) $\mathrm{AD}$ or flares (presence of symptoms intermittent with periods of remission); (3) concomitant medication; (4) VAS score for itch; (5) VAS score for sleep loss; (6) change in AD was qualitatively assessed by the physician in consultation with the parents as "very much improved," "much improved," "no change," and "worse"; and (7) adverse events. Adherence to the synbiotic was calculated based on the patient's diary and categorized as good $(\geq 80 \%)$ or poor $(<80 \%)$. Patients were assessed at baseline and at the end of treatment (8 weeks).

\section{Statistical analysis}

Based on the results of a randomized double-blind controlled trial carried out in a similar population of children with $\mathrm{AD}$ and in which a mean (standard deviation, SD) reduction of SCORAD of 39.2 (24.22) was found after 8 weeks of treatment with a synbiotic mixture [9], it was estimated that a sample of 867 patients would be needed to detect a mean change of 39.2 points in the SCORAD index, accepting a SD of 24.22 and a level of precision of 1.7 for a two-tailed analysis with an alpha error of 0.05 . A lost to follow-up rate of $10 \%$ was assumed.

Analysis of the primary efficacy variable was performed in the intention-to-treat (ITT) data set, which included all patients treated with the synbiotic product independently of the level of adherence, and in the per-protocol (PP) data set, which included all patients who completed the study with a level of adherence of $\geq 80 \%$. In the ITT analysis, the last observation carried forward was used for missing data. Categorical variables are expressed as frequencies and percentages, and continuous variables as mean and SD or median and interquartile range, with the corresponding 95\% confidence interval (CI). The chi-square test, the Fisher's exact probability test, or the McNemar's test were used for the comparison of categorical variables, and the Student's $t$ test, the Mann-Whitney $U$ test, or the Wilcoxon signed-rank test for the comparison of continuous variables according to normal or non-normal distribution of data. Agreement between variables was analyzed using kappa statistics. Variables independently associated with improvement of $\mathrm{AD}$ (expressed as difference between baseline and final SCORAD) were analyzed in a multiple linear regression model, in which all variables with a $P$ value $<0.200$ in bivariate analyses were included. Statistical significance was set at $P<0.05$. The Statistical Package for the Social Sciences (SPSS Inc., Chicago, IL, USA) v22 was used for statistical analysis.

\section{Results}

The rate of recruitment was lower than expected, and a total of 353 patients were recruited within the foreseen period. Thirtythree $(9.3 \%)$ patients did not meet the selection criteria and were excluded. Therefore, the study population included 320 patients, of which 275 completed the study. The flow diagram of participants is shown in Fig. 1.

Baseline characteristics are summarized in Table 1. Most $(54.9 \%)$ patients were girls, and the median age was 4 years (range 3 months to 11.8 years). Concomitant respiratory allergy and food allergy were reported in $36.9 \%$ and $21.3 \%$ of patients, respectively; history of parental atopy was present in $70.9 \%$ of cases, most frequently $\mathrm{AD}$ or respiratory allergy. The median duration of AD was 3.4 years (IQR 1.7-6.2 years). Persistent AD was present in $52.1 \%$ of patients. Less than half of patients $(43.4 \%)$ were receiving concomitant treatments, most frequently topical corticosteroids $(21.3 \%)$, antihistamines $(19.7 \%)$, or treatments for concomitant diseases ("Others," Table 1). Thirty-three (11.1\%) patients had changes in their concomitant treatment between the baseline and the follow-up visits.

At baseline, the mean (SD) SCORAD was 45.5 (15.5) (95\% CI 43.8-47.3) (Table 2), and more than half of the patients $(57.3 \%)$ had moderate disease (SCORAD 25-50) (Fig. 2). The intensity of the cutaneous symptoms was moderate in most patients. The mean VAS score for pruritus was 5.7 (2.2) (95\% CI 5.5-5.9) and for sleep loss 3.1 (2.5) (95\% CI 2.9-3.4). At the final visit (week 8), the mean SCORAD was 19.4 (14.6) (95\% CI 17.7-21.2), cutaneous lesions had cleared or were of mild intensity in most patients, and the mean VAS score for pruritus was 2.3 (2.2) (95\% CI 2.0-2.5) and for sleep loss 1.1 (1.8) (95\% CI 0.9-1.3). Average (SD) intra-patient difference in SCORAD score between the basal and the follow-up visits was 27.0 (15.1) points (median 27.0; range -6 to 79.3). All differences in SCORAD were statistically

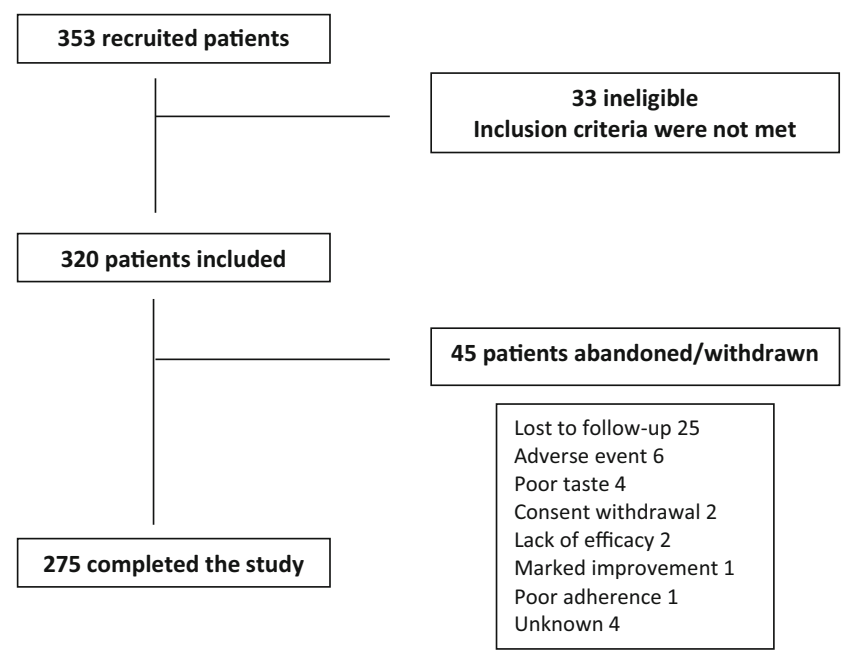

Fig. 1 Flow diagram of participants in the study 
Table 1 Baseline characteristics of 320 children with atopic dermatitis (AD)

\begin{tabular}{|c|c|c|}
\hline Variables & Number $(\%)$ & Mean (SD) \\
\hline \multicolumn{3}{|l|}{ Sex } \\
\hline Girls & $175(54.9)$ & \\
\hline Age, years & & $5.1(3.1)$ \\
\hline$<2$ years of age & $43(13.4)$ & \\
\hline$\geq 2$ and $<5$ years of age & $122(38.1)$ & \\
\hline$\geq 5$ years of age & $155(48.4)$ & \\
\hline \multicolumn{3}{|l|}{ Parental history } \\
\hline \multicolumn{3}{|l|}{ Education level, $n=304$} \\
\hline No studies & $15(4.9)$ & \\
\hline Primary education & $37(12.2)$ & \\
\hline Secondary education & 109 (35.9) & \\
\hline University degree & $143(47.0)$ & \\
\hline Atopy, present & $227(70.9)$ & \\
\hline $\mathrm{AD}$ & $145(45.3)$ & \\
\hline Respiratory allergy & $117(36.6)$ & \\
\hline Food allergy & $36(11.3)$ & \\
\hline Drug allergy & $25(7.8)$ & \\
\hline Living with pets & $73(22.8)$ & \\
\hline Respiratory allergy, $n=309$ & $114(36.9)$ & \\
\hline Food allergy, $n=301$ & $64(21.3)$ & \\
\hline Drug allergy, $n=302$ & $5(1.7)$ & \\
\hline Duration of $\mathrm{AD}$, years & & $4.0(3.0)$ \\
\hline \multicolumn{3}{|l|}{ Type of disease, $n=292$} \\
\hline Persistent & $152(52.1)$ & \\
\hline With flares & $140(47.9)$ & \\
\hline Flares per month & & $1.9(1.3)$ \\
\hline Concomitant treatment* & $139(43.4)$ & \\
\hline Systemic antibiotics & $11(3.4)$ & \\
\hline Topical antibiotics & $9(2.8)$ & \\
\hline Antihistamines & $63(19.7)$ & \\
\hline Systemic corticosteroids & $7(2.2)$ & \\
\hline Topical corticosteroids & $68(21.3)$ & \\
\hline Topical immunosuppressants & $12(3.89)$ & \\
\hline Other & $71(22.2)$ & \\
\hline
\end{tabular}

*A patient could have more than one concomitant treatment

significant $(P<0.001)$ (Table 2$)$. The number of patients with severe disease decreased from $35.1 \%$ at baseline to $4.4 \%$ at week 8 (Fig. 2). In the PP dataset, the mean SCORAD index decreased from $47.3(15.5)(95 \%$ CI 45.3-49.3) at baseline to 19.2 (14.8) (95\% CI 17.3-21.1) after 8 weeks of treatment $(P<0.001)$.

The percentage of physicians rating $\mathrm{AD}$ as "very much improved," "much improved," "no change," and "worse" was $35.9 \%, 49.8 \%, 13.9 \%$, and $0.3 \%$, respectively. These percentages were similar to the parents' opinion $(38.9 \%, 42.7 \%$, $17.4 \%$, and $1.0 \%$, respectively) (kappa 0.673 ). The kappa index for concordance between improvement/no improvement and $30 \%$ and $50 \%$ improvement of the SCORAD was 0.698 and 0.443 for physicians, and 0.643 and 0.520 for the parents.

Adherence to the synbiotic product was $\geq 80 \%$ in $89.7 \%$ of patients. The decrease in the SCORAD index was greater in patients with higher adherence (28.1 (14.9) points in adherent patients versus $18.0(14.6)$ points in non-adherent patients $(P=0.004))$.

In multiple linear regression analysis, the independent predictors of stronger improvement (that is, a greater decrease in the SCORAD index between baseline visit and week 8) were higher baseline SCORAD (OR 0.51, 95\% CI 0.41-0.61; $P=$ 0.0001 ) and adherence $\geq 80 \%$ (OR 7.29, 95\% CI 1.85-12.73; $P=0.009$ ) (Table 3).

A total of 29 adverse events were recorded in 21 patients (Table 4). Of them, only two events of abdominal pain observed in the same patient were judged by the investigator to be potentially related to the product.

\section{Discussion}

This study carried out in real-world practice shows that an oral supplementation with a synbiotic product with high levels of viable organisms for 8 weeks was effective in improving $\mathrm{AD}$ in children. The product included a mixture of four bacterial strains of commensal organisms (L. casei, L. rhamnosus, L. plantarum, and B. lactis), fructooligosaccharide, galactooligosaccharide, and biotin, the benefits of which in several immune-mediated and allergic diseases has been documented $[15,19,23,25]$. Importantly, improvement was observed in long-standing $\mathrm{AD}$ (mean time from diagnosis of 4 years), both in persistent disease and in $\mathrm{AD}$ with flares, in patients with and without concomitant atopies, as well as in the presence or absence of underlying treatment for AD. These variables did not affect improvement of SCORAD index in bivariate analyses. Treatment was well tolerated, and of the 29 registered adverse events, only two events of abdominal pain were judged to be possibly related to the synbiotic product.

We used the SCORAD index, a valid and reliable tool, for the measurement of the main outcome of the study [22]. Statistically significant improvements in the total score as well as in the intensity of six cutaneous symptoms, pruritus, and sleepiness were found after 8 weeks of treatment. Also, the level of agreement of physicians and parents regarding amelioration of $\mathrm{AD}$ was substantial for a $30 \%$ decrease of the SCORAD score and moderate for a $50 \%$ decrease.

Conflicting data on the effect of probiotics on $\mathrm{AD}$ have been reported in literature, although in general, results favor probiotics over controls. A meta-analysis of $25 \mathrm{RCTs}$ with 1599 AD patients found a greater improvement in SCORAD in the probiotics group compared to controls in children 1 to 
Table 2 Differences in results of the SCORAD index between baseline and the final visit after 8 weeks of treatment with the probiotic product

\begin{tabular}{|c|c|c|c|}
\hline Variable & Baseline & Final visit (week 8) & $P$ value \\
\hline SCORAD score, mean (SD) $(95 \% \mathrm{CI})$ & $45.5(15.5)(43.8-47.3)$ & $19.4(14.6)(17.7-21.2)$ & $<0.001^{*}$ \\
\hline \multicolumn{4}{|l|}{ Cutaneous symptoms, $\%$ of patients } \\
\hline Erythema & & & $<0.001^{\dagger}$ \\
\hline None & 3.0 & 32.4 & \\
\hline Mild & 23.3 & 52.7 & \\
\hline Moderate & 58.5 & 12.2 & \\
\hline Severe & 15.3 & 2.7 & \\
\hline Edema & & & $<0.001^{\dagger}$ \\
\hline None & 39.4 & 74.6 & \\
\hline Mild & 35.5 & 18.1 & \\
\hline Moderate & 21.1 & 6.0 & \\
\hline Severe & 3.9 & 1.2 & \\
\hline Oozing/crusting & & & $<0.001^{\dagger}$ \\
\hline None & 36.2 & 75.1 & \\
\hline Mild & 28.3 & 19.3 & \\
\hline Moderate & 26.4 & 4.0 & \\
\hline Severe & 9.1 & 1.6 & \\
\hline Excoriation & & & $<0.001^{\dagger}$ \\
\hline None & 18.1 & 59.9 & \\
\hline Mild & 32.1 & 29.8 & \\
\hline Moderate & 36.9 & 7.9 & \\
\hline Severe & 12.9 & 2.4 & \\
\hline Skin thickening (lichenification) & & & $<0.001^{\dagger}$ \\
\hline None & 42.1 & 64.3 & \\
\hline Mild & 27.4 & 27.0 & \\
\hline Moderate & 22.6 & 7.9 & \\
\hline Severe & 7.9 & 0.8 & \\
\hline Dryness & & & $<0.001^{\dagger}$ \\
\hline None & 1.7 & 15.6 & \\
\hline Mild & 11.4 & 55.5 & \\
\hline Moderate & 52.0 & 24.0 & \\
\hline Severe & 34.9 & 4.9 & \\
\hline Pruritus, VAS score, mean (SD) (95\% CI) & $5.7(2.2)(5.5-5.9)$ & $2.3(2.2)(2.0-2.5)$ & $<0.001 *$ \\
\hline Sleepiness, VAS score, mean (SD) (95\% CI) & $3.1(2.5)(2.9-3.4)$ & $1.1(1.8)(0.9-1.3)$ & $<0.001 *$ \\
\hline
\end{tabular}

*Wilcoxon signed-rank test; ${ }^{\dagger}$ McNemar's test
18 years old (difference in change in SCORAD -5.74 points vs control, $95 \%$ confidence interval -7.27 to -4.20 ), and in adults (difference in change in SCORAD - 8.26 points vs control, $95 \%$ confidence interval -13.28 to -3.25 ), although the effect was not proven in infants ( $<1$ year old) [12]. Similarly, a meta-analysis on RCT on synbiotics in children with AD (6 studies, 369 children) found a greater decrease in SCORAD (by 6.56 points) in synbiotic groups vs placebo (95\% CI, -11.43 to -1.68 ) [6]. Heterogeneity was high in both meta-analyses, and in both cases, the authors found greater benefits in case of treatment with mixed bacterial species [6, $12]$.
Yesilova et al. [24] carried out a study in 40 children with AD who were randomized to supplementation with a probiotic mixture or placebo. Similarly, these patients were treated for 8 weeks with a high-dose $\left(2 \times 10^{9} \mathrm{CFU}\right)$ multistrain probiotic complex containing B. bifidum, L. acidophilus, L. casei, and L. salivarius. The authors observed a significant decrease of SCORAD values at the end of treatment, with the magnitude of SCORAD reduction (mean change from baseline 23.0 points, compared to 12.8 points in placebo arm) comparable to that in our study (mean change from baseline 26.1 points). The probiotic supplement was also effective for reducing serum IL-5, IL-6, IFN- $\gamma$, and total serum IgE levels; 


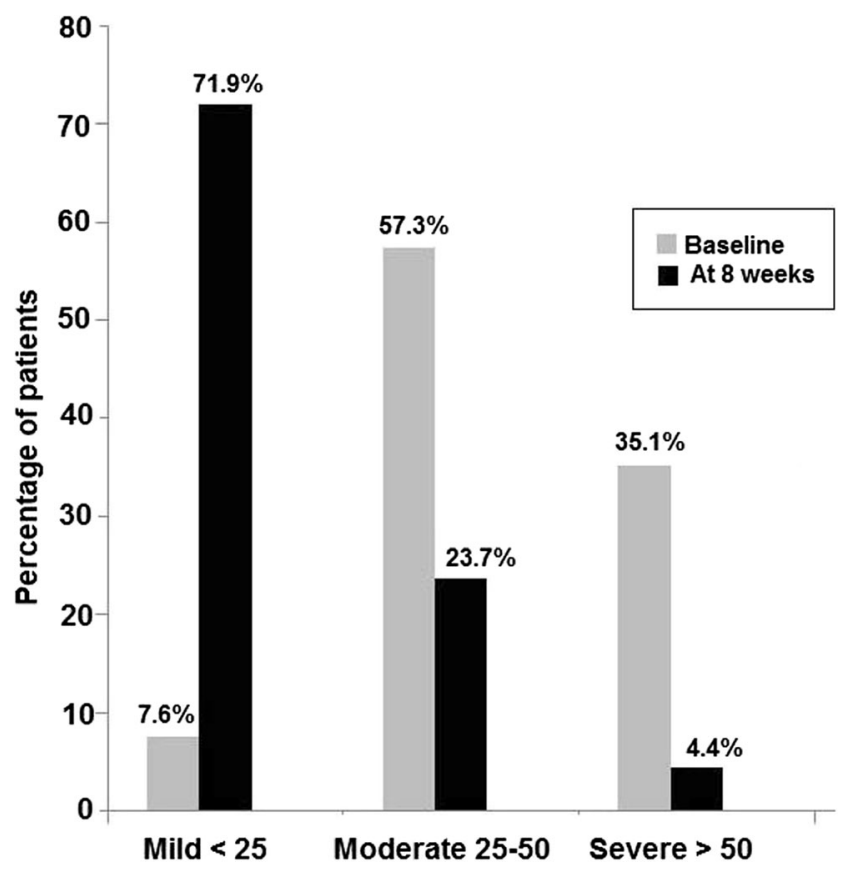

Fig. 2 Changes in SCORAD scores between baseline and after 8 weeks of treatment with the synbiotic product. Differences for mild, moderate, and severe disease are statistically significant $(P<0.001)$ unfortunately, the effect on IgE levels and proinflammatory cytokines was not examined in our study.

The improvement in SCORAD index and in all symptoms that was observed in our study was stronger than in most published controlled trials on probiotics in AD, which could be due to various reasons, including differences in study design, dose and strains of probiotics used, duration of intervention, characteristics of the study population, and the sample size. Since this was a single-arm, non-controlled study, we cannot rule out the placebo effect and/or improvement due to the natural course of the disease. It should be noted, however, that many participants had a long-standing AD (mean time since diagnosis 4 years), and about half had persistent disease, so it seems unlikely that all the improvement was due to the natural progression of the disease. Besides, since this was an observational study, there were no restrictions on concomitant medication, and $21 \%$ of study participants were receiving topical corticosteroids and $19 \%$ antihistamines. Overall, however, the percentage of patients receiving medical treatment for $\mathrm{AD}$ was lower than what could be expected in this population (most patients with moderate or even severe disease), which suggests that $\mathrm{AD}$ in our practice may be undertreated. Importantly, patients with and without

Table 3 Summary of bivariate analyses and multiple regression analysis. The dependent variable was the intra-patient change in SCORAD index

\begin{tabular}{|c|c|c|c|c|c|c|c|}
\hline \multirow[t]{2}{*}{ Variable } & \multicolumn{2}{|c|}{ Non-adjusted coefficients } & \multirow[t]{2}{*}{$P$} & \multirow{2}{*}{$\begin{array}{l}\text { Adjusted coefficients } \\
\text { Beta }\end{array}$} & \multirow[t]{2}{*}{$t$} & \multicolumn{2}{|l|}{$95 \% \mathrm{CI}$ for $\mathrm{B}$} \\
\hline & $\mathrm{B}$ & SD & & & & Lower limit & Upper limit \\
\hline Age & 0.053 & 0.296 & 0.859 & 0.011 & 0.178 & -0.529 & 0.635 \\
\hline Sex & -1.783 & 1.862 & 0.339 & -0.059 & -0.958 & -5.449 & 1.882 \\
\hline History of AD in parents & 0.465 & 1.924 & 0.809 & 0.016 & 0.242 & -3.324 & 4.254 \\
\hline History of food allergy in parents & -2.393 & 2.941 & 0.417 & -0.054 & -0.814 & -8.188 & 3.403 \\
\hline History of respiratory allergy in parents & 2.926 & 1.981 & 0.141 & 0.094 & 1.477 & -0.976 & 6.827 \\
\hline History of drug allergy in parents & 4.890 & 3.289 & 0.138 & 0.099 & 1.487 & -1.591 & 11.371 \\
\hline Living with pets & -0.596 & 2.195 & 0.786 & -0.017 & -0.272 & -4.921 & 3.728 \\
\hline Parents' education level & -2.797 & 1.103 & 0.012 & -0.157 & -2.536 & -4.968 & -0.625 \\
\hline History of respiratory allergy in patient & 1.853 & 1.944 & 0.341 & 0.059 & 0.953 & -1.974 & 5.681 \\
\hline History of food allergy in patient & 4.464 & 2.270 & 0.050 & 0.123 & 1.967 & -0.006 & 8.935 \\
\hline History of drug allergy in patient & 4.032 & 7.611 & 0.597 & 0.033 & 0.530 & -10.955 & 19.020 \\
\hline Duration of $\mathrm{AD}$ & 0.401 & 0.319 & 0.210 & 0.082 & 1.256 & -0.228 & 1.031 \\
\hline Age at $\mathrm{AD}$ diagnosis & -0.495 & 0.633 & 0.435 & -0.052 & -0.782 & -1.743 & 0.752 \\
\hline Type of AD (persistent vs with flares) & -2.218 & 1.921 & 0.249 & -0.074 & -1.154 & -6.002 & 1.566 \\
\hline Basal SCORAD score & 0.530 & 0.050 & $<0.001$ & 0.546 & 10.623 & 0.432 & 0.628 \\
\hline Season of start of treatment (summer vs spring)* & -0.545 & 0.852 & 0.523 & -0.040 & -0.640 & -2.222 & 1.132 \\
\hline Adherence to probiotic $(<80 \%$ vs $\geq 80 \%)$ & 10.059 & 3.247 & 0.002 & 0.190 & 3.098 & 3.664 & 16.454 \\
\hline Pre-existent concomitant treatment & 0.766 & 2.884 & 0.791 & 0.024 & 0.265 & -4.943 & 6.474 \\
\hline De novo concomitant treatment & 3.487 & 2.860 & 0.225 & 0.109 & 1.219 & -2.174 & 9.149 \\
\hline Any concomitant treatment & 1.621 & 1.848 & 0.381 & 0.054 & 0.877 & -2.018 & 5.261 \\
\hline \multicolumn{8}{|c|}{ Best-fitting model } \\
\hline Basal SCORAD score & 0.508 & 0.050 & $<0.001$ & 0.528 & 10.103 & 0.409 & 0.608 \\
\hline Adherence to probiotic $(<80 \%$ vs $\geq 80 \%)$ & 7.289 & 2.762 & 0.009 & 0.138 & 2.639 & 1.850 & 12.728 \\
\hline
\end{tabular}


Table 4 Details of 29 adverse events registered in 21 patients

\begin{tabular}{lcl}
\hline Adverse event & No. events & Causality with the synbiotic product \\
\hline Cutaneous & 10 & \\
Worsening of dermatitis & 2 & Unrelated \\
Superinfection & 2 & Unrelated \\
Mollusculum contagiosum & 2 & Unrelated \\
Eczema & 1 & Unrelated \\
Erythema and scaling & 1 & Unrelated \\
Edema and pruritus & 1 & Unrelated \\
Pruritus and scaling & 1 & Unrelated \\
Respiratory & 9 & \\
Bronchospasm & 4 & Unrelated \\
Upper tract infection & 4 & Unrelated \\
Asthma attack & 1 & Unrelated \\
Gastrointestinal & 7 & Unrelated \\
Acute gastroenteritis & 4 & Possibly related in 1 \\
Abdominal pain & 2 & Unrelated \\
Vomiting & 1 & Unrelated \\
Neurological & 2 & Unrelated \\
Irritability and insomnia & 1 & \\
Nervousness, irritability, and insomnia & 1 & \\
\hline
\end{tabular}

concomitant medication showed a similar improvement in $\mathrm{AD}$, suggesting that the observed changes could not be attributed exclusively to concomitant treatments. Furthermore, we found a significant association between the adherence to treatment with the multistrain high-dose synbiotic product and decrease of SCORAD index. Good adherence was the most important predictive factor of clinical improvement both in the bivariate and multivariate analyses, which further supports the idea that at least part of the observed improvement in AD was due to the synbiotic treatment.

One possible limitation affecting the validity of the results was that the participant rate was lower than the projected sample size. The observational character of the study allowed including a varied sample of patients, and the multivariate analysis allowed assessing the effectiveness of treatment in relation to patient's characteristics. Well-designed RCTs should be conducted to elucidate the effectiveness of synbiotics in AD treatment. Including more representative real-world patient samples and complementing RCT with data from observational studies should help physicians treating children with $\mathrm{AD}$ to have appropriate evidence on which to base their clinical decisions in daily practice.

In conclusion, our results indicate that supplementation with multistrain high-dose synbiotics (L. casei, L. rhamnosus, L. plantarum, and B. lactis, combined with fructooligosaccharides, galactooligosaccharides, and biotin) improves $\mathrm{AD}$ in children, whereas the tolerability and safety profile are very good. Further studies, including RTCs, are needed to add evidence on the benefits of synbiotics in the treatment of pediatric $\mathrm{AD}$ in real-life clinical practice.

Acknowledgments The authors thank all the investigators, patients, and their parents who participated in the study; Marta Pulido, MD, $\mathrm{PhD}$, for assistance in writing the manuscript; Zhanna Shcheprova, $\mathrm{PhD}$, for the help in study supervision and manuscript preparation; and Francisco López de Saro, PhD (Trialance SCCL), for medical writing support.

Authors' contributions MDI, PRR, DGA, and VVI contributed to study design, data analysis, and interpretation, and drafted the article.

Funding This study was sponsored by Chiesi Spain S.A.U. The study product (Produo Derma ${ }^{\circledR}$ ) was provided by Chiesi Spain S.A.U.

\section{Compliance with ethical standards}

The study was conducted in accordance with the Declaration of Helsinki (7th revision), the Spanish regulations on observational studies (Order SAS 3470/2009) and Spanish personal data protection law (Law 15/ 1999). The study protocol was approved by the Ethics Committee of Hospital Infantil Universitario Niño Jesús, Madrid, Spain. Parents or legal representatives of all patients gave written informed consent before inclusion. All data were anonymized.

Conflict of interest MDI received research funding from the Health Research Fund of the Carlos III Health Institute, and from Aimmune Therapeutics; serves as a consultant for Merk, Novartis, and Chiesi Spain; and has received payments for lectures from the Spanish Society of Allergology and Clinical Immunology, Leti Pharma, Merck, FAES Pharma, and MSD.

PRR received research funding from the Health Research Fund of Carlos III Health Institute, the Foundation for Biomedical Research of the Niño Jesús University Children's Hospital, the Spanish Society of 
Allergology, and the Clinical Immunology Foundation. He received honoraria for consultancy and/or advisory board and/or lectures from ALKAbelló, HAL-Allergy, FAES Pharma, LETI Pharma, Merck, Aimmune Therapeutics, Allergy Therapeutics, MEDA Pharma, and Novartis.

DGA and VVI are employees of Chiesi Spain.

Informed consent Parents or legal representatives of all patients included in the study gave written informed consent before inclusion.

Open Access This article is distributed under the terms of the Creative Commons Attribution 4.0 International License (http:// creativecommons.org/licenses/by/4.0/), which permits unrestricted use, distribution, and reproduction in any medium, provided you give appropriate credit to the original author(s) and the source, provide a link to the Creative Commons license, and indicate if changes were made.

\section{References}

1. Ben-Gashir MA (2003) Relationship between quality of life and disease severity in atopic dermatitis/eczema syndrome during childhood. Curr Opin Allergy Clin Immunol 3:369-373

2. Betsi GI, Papadavid E, Falagas ME (2008) Probiotics for the treatment or prevention of atopic dermatitis: a review of the evidence from randomized controlled trials. Am J Clin Dermatol 9:93-103

3. Boyle RJ, Bath-Hextall FJ, Leonardi-Bee J, Murrell DF, Tang ML (2009) Probiotics for the treatment of eczema: a systematic review. Clin Exp Allergy 39:1117-1127

4. Cao L, Wang L, Yang L, Tao S, Xia R, Fan W (2015) Long-term effect of early life supplementation with probiotics on preventing atopic dermatitis: a meta-analysis. J Dermatol Treat 26:537-540

5. Carr WW (2013) Topical calcineurin inhibitors for atopic dermatitis: review and treatment recommendations. Paediatr Drugs 15: 303-310

6. Chang YS, Trivedi MK, Jha A, Lin YF, Dimaano L, GarcíaRomero MT (2016) Synbiotics for prevention and treatment of atopic dermatitis: a meta-analysis of randomized controlled trials. JAMA Pediatr 170:236-242

7. da IPN CB, Accioly E, de Carvalho Padilha P (2013) Effect of the use of probiotics in the treatment of children with atopic dermatitis; a review. Nutr Hosp 28:16-26

8. de Vrese M, Schrezenmeir J (2008) Probiotics, prebiotics, and synbiotics. Adv Biochem Eng Biotechnol 111:1-66

9. Farid R, Ahanchian H, Jabbari F, Moghiman T (2011) Effect of a new synbiotic mixture on atopic dermatitis in children: a randomized-controlled trial. Iran J Pediatr 21:225-230

10. Galli E, Neri I, Ricci G, Baldo E, Barone M, Belloni Fortina A, Bernardini R, Berti I, Caffarelli C, Calamelli E, Capra L, Carello R, Cipriani F, Comberiati P, Diociaiuti A, El Hachem M, Fontana E, Gruber M, Haddock E, Maiello N, Meglio P, Patrizi A, Peroni D, Scarponi D, Wielander I, Eichenfield LF (2016) Consensus conference on clinical management of pediatric atopic dermatitis. Ital J Pediatr 42:26

11. Hengge UR, Ruzicka T, Schwartz RA, Cork MJ (2016) Adverse effects of topical glucocorticosteroids. J Am Acad Dermatol 54:115

12. Kim SO, Ah YM, Yu YM, Choi KH, Shin WG, Lee JY (2014) Effects of probiotics for the treatment of atopic dermatitis: a metaanalysis of randomized controlled trials. Ann Allergy Asthma Immunol 113:217-226

13. Kobayashi T, Glatz M, Horiuchi K, Kawasaki H, Akiyama H, Kaplan DH, Kong HH, Amagai M, Nagao K (2015) Dysbiosis and Staphylococcus aureus colonization drives inflammation in atopic dermatitis. Immunity 42:756-766

14. Krakowski AC, Eichenfield LF, Dohil MA (2008) Management of atopic dermatitis in the pediatric population. Pediatrics 122:812824

15. Liu J, Chen FH, Qiu SQ, Yang LT, Zhang HP, Liu JQ, Geng XR, Yang G, Liu ZQ, Li J, Liu ZG, Li HB, Yang PC (2016) Probiotics enhance the effect of allergy immunotherapy on regulating antigen specific B cell activity in asthma patients. Am J Transl Res 8:52565270

16. Matsumoto M, Aranami A, Ishige A, Watanabe K, Benno Y (2007) LKM512 yogurt consumption improves the intestinal environment and induces the T-helper type 1 cytokine in adult patients with intractable atopic dermatitis. Clin Exp Allergy 37:358-370

17. Nonaka Y, Izumo T, Izumi F, Maekawa T, Shibata H, Nakano A, Kishi A, Akatani K, Kiso Y (2008) Antiallergic effects of Lactobacillus pentosus strain S-PT84 mediated by modulation of Th1/Th2 immunobalance and induction of IL-10 production. Int Arch Allergy Immunol 145:249-257

18. Nutten S (2016) Atopic dermatitis: global epidemiology and risk factors. Ann Nutr Metab 66(Suppl 1):8-16

19. Prakash S, Tomaro-Duchesneau C, Saha S, Rodes L, Kahouli I, Malhotra M (2014) Probiotics for the prevention and treatment of allergies, with an emphasis on mode of delivery and mechanism of action. Curr Pharm Des 20:1025-1037

20. Raimer SS (2000) Managing pediatric atopic dermatitis. Clin Pediatr 39:1-14

21. Rather IA, Bajpai VK, Kumar S, Lim J, Paek WK, Park YH (2016) Probiotics and atopic dermatitis: an overview. Front Microbiol 7: 507

22. Severity scoring of atopic dermatitis: the SCORAD index (1993) Consensus Report of the European Task Force on Atopic Dermatitis. Dermatology 183:23-31

23. Vitaliti G, Pavone P, Guglielmo F, Spataro G, Falsaperla R (2014) The immunomodulatory effect of probiotics beyond atopy: an update. J Asthma 51:320-332

24. Yeșilova Y, Çalka Ö, Akdeniz N, Berktaș M (2012) Effect of probiotics on the treatment of children with atopic dermatitis. Ann Dermatol 24:189-193

25. Zajac AE, Adams AS, Turner JH (2015) A systematic review and meta-analysis of probiotics for the treatment of allergic rhinitis. Int Forum Allergy Rhinol 5:524-532 